PROCEEDINGS OF THE

AMERICAN MATHEMATICAL SOCIETY

Volume 127, Number 2, February 1999, Pages 355-361

S 0002-9939(99)04548-7

\title{
THE NONQUADRATIC IMAGINARY CYCLIC FIELDS OF 2-POWER DEGREES WITH CLASS NUMBERS EQUAL TO THEIR GENUS CLASS NUMBERS
}

\author{
STÉPHANE LOUBOUTIN
}

(Communicated by William W. Adams)

\begin{abstract}
It is known that there are only finitely many imaginary abelian number fields with class numbers equal to their genus class numbers. Here, we determine all the imaginary cyclic fields of 2-power degrees with class numbers equal to their genus class numbers.
\end{abstract}

\section{INTRODUCTION}

Let $K$ be an abelian number field of degree $n_{K}$. The narrow genus field $G_{K}$ of $K$ is the maximal abelian number field containing $K$ and unramified above $K$ at all the finite places. Note that if $K$ is imaginary, then $G_{K}$ is an imaginary abelian number field and $G_{K} / K$ is unramified at all the places. According to class field theory $G_{K}$ is included in the narrow Hilbert class field $H_{K}^{+}$of $K$ and the degree $g_{K}=\left[G_{K}: K\right]$ divides the narrow class number $h_{K}^{+}$of $K$. In particular, if $K$ is imaginary, then $g_{K}$ divides $h_{K}$ the class number of $K$. When the group $X_{K}$ of Dirichlet characters associated with an abelian number field $K$ is given, we can easily compute the degree of $G_{K}$ and the genus class number $g_{K}$ of $K$ : we have $g_{K}=\frac{1}{n_{K}} \prod_{p} e_{p}$ where this product ranges over all the rational primes $p$ which are ramified in $K$ and where $e_{p}$ denotes the index of ramification of $p$ in the extension $K / Q$ (see Chapter 3 in [Wa]). In [Lou2] we proved that there are only finitely many imaginary abelian number fields such that their class numbers $h_{N}$ are equal to their genus class numbers $g_{N}$ and proved that apart from the quadratic and bicyclic quadratic ones, one can find an effective upper bound on their conductors. The aim of this paper is to determine all the imaginary cyclic fields of 2-power degrees with class numbers equal to their genus class numbers. There are 28 such number imaginary cyclic fields:

Theorem 1. There are 23 imaginary cyclic quartic fields with class numbers equal to their genus class numbers. Their conductors are less than or equal to 255 and their class numbers are less than or equal to 8. They are listed in Table 1 of Section 3. There are 4 imaginary cyclic octic fields with class numbers equal to their genus class numbers. Their conductors are less than or equal to 85 and their class numbers are less than or equal to 4. They are listed in Table 2 of Section 3. There is 1

Received by the editors January 31, 1997 and, in revised form, May 28, 1997.

1991 Mathematics Subject Classification. Primary 11R20, 11R29; Secondary $11 \mathrm{R} 42$.

Key words and phrases. Genus field, relative class number, class number, cyclic number field.

(C)1999 American Mathematical Society 
imaginary cyclic field of degree sixteen with class numbers equal to its genus class numbers: the cyclotomic field $Q\left(\zeta_{17}\right)$ which has class number one. Finally, there is no imaginary cyclic field of 2-power degree $2^{m} \geq 32$ with class number equal to its genus class number.

To prove this theorem we first use lower bounds on relative class numbers of imaginary cyclic fields of 2-power degrees $2^{m} \geq 4$ (see Theorem 5 ). Second, according to Lemma 2, relative class numbers of imaginary cyclic fields of 2-power degrees $2^{m} \geq 4$ with class numbers equal to their genus class numbers cannot be that large. Hence, we will thirdly get bounds on the conductors of the imaginary cyclic fields of 2-power degrees $2^{m} \geq 4$ with class numbers equal to their genus class numbers (see Corollary 6 ). Fourth, we will compute the relative class numbers of all the possible imaginary cyclic fields of 2 -power degrees $2^{m} \geq 4$ of conductors less than or equal to these bounds. This will provide us with a short list of imaginary cyclic fields of 2-power degrees $2^{m} \geq 4$ of conductors less than or equal to these bounds and with relative class numbers satisfying the necessary condition of Lemma 2. Finally, we will determine all the fields of this small list which are such that their class numbers are equal to their genus class numbers.

Lemma 2. Let $N$ be an imaginary abelian number field of degree $2 n$ with maximal real subfield $N^{+}$. Let $t$ denote the number of rational primes $p$ such that all the ideals of $N^{+}$above $p$ are ramified in the quadratic extension $N / N^{+}$. If $h_{N}=g_{N}$, then $h_{N}^{-}=2^{t-1+\epsilon}$, where $\epsilon=0$ or 1 according as $G_{N^{+}}$the narrow genus field of $N^{+}$is real or imaginary.

Therefore, if $N$ is an imaginary cyclic field of 2-power degree $2^{m} \geq 2$ and if $h_{N}=g_{N}$, then $h_{N}^{-}=2^{t-1}$ where $t$ denotes the number of prime divisors of $f_{N}$, the conductor of $N$.

Proof. Since $h_{N}=g_{N}$, then $H_{N}=G_{N}$. Let $H_{N^{+}}^{+}$denote the narrow Hilbert class field of $N^{+}$. Since $H_{N^{+}}^{+} / N^{+}$is an abelian extension unramified at all the finite places and since $N$ is imaginary, then $H_{N^{+}}^{+} N / N$ is an abelian extension unramified at all the places. Therefore, we have $H_{N^{+}}^{+} N \subseteq H_{N}=G_{N}$ and $H_{N^{+}}^{+}$is an abelian field. Hence, $H_{N^{+}}^{+}=G_{N^{+}}$.

Now, if $G_{N^{+}}$is real, we have $H_{N^{+}}=G_{N^{+}}$and we get $h_{N^{+}}=\left[H_{N^{+}}: N^{+}\right]=$ $\left[G_{N^{+}}: N^{+}\right]=\frac{1}{n} \prod_{p} e_{p}^{+}$which together with $h_{N}=g_{N}=\frac{1}{2 n} \prod_{p} e_{p}$ yields

$$
h_{N}^{-}=\frac{1}{2} \prod_{p}\left(e_{p} / e_{p}^{+}\right)
$$

and the desired result. In the same way, if $G_{N^{+}}$is imaginary and if we let $G_{N^{+}}^{+}$ denote its maximal real subfield, then $H_{N^{+}}=G_{N^{+}}^{+}$and we get $h_{N^{+}}=\left[H_{N^{+}}\right.$: $\left.N^{+}\right]=\left[G_{N^{+}}^{+}: N^{+}\right]=\frac{1}{2}\left[G_{N^{+}}: N^{+}\right]=\frac{1}{2 n} \prod_{p} e_{p}^{+}$which together with $h_{N}=g_{N}=$ $\frac{1}{2 n} \prod_{p} e_{p}$ yields

$$
h_{N}^{-}=\prod_{p}\left(e_{p} / e_{p}^{+}\right)
$$

and the desired result.

\section{IMAGINARY CYCLIC FIELDS OF 2-POWER DEGREES}

Let $N, f_{N}, h_{N}, N^{+}, f_{N^{+}}$and $h_{N^{+}}$denote a cyclic field of 2-power degree $2^{m} \geq 4$, its conductor, its class number, its only subfield of degree $2^{m-1}$ and the conductor 
and class number of $N^{+}$, respectively. Let $\chi_{N}$ be a primitive Dirichlet character of order $2^{m}$ associated with $N$. Then $N$ is real or imaginary according as $\chi_{N}(-1)=$ +1 or $\chi_{N}(-1)=-1$. Now, assume $N$ is imaginary. Then $N^{+}$is real, $h_{N^{+}}$divides $h_{N}$ and we define the relative class number $h_{N}^{-}$of $N$ to be $h_{N} / h_{N^{+}}$. Moreover, let $w_{N}$ denote the number of roots of unity in $N$. Then $w_{N}=2$ except if $p=2 m+1$ is prime and $N=Q\left(\zeta_{p}\right)$, in which case $w_{N}=2 p$ (see [Lou1, Lemma (b)]). Since $N / Q$ is cyclic, its Hasse unit index $Q_{N}$ is equal to 1 and

$$
h_{N}^{-}=w_{N} \prod_{j=0}^{2^{m-2}-1}\left|\frac{1}{2\left(2-\overline{\chi_{N}^{2 j+1}}(2)\right)} \sum_{1 \leq x \leq f_{N} / 2} \chi_{N}^{2 j+1}(x)\right|^{2}
$$

(see [Wa, Theorem 4.17] and [Lou1]). Now we explain how we construct the imaginary cyclic fields of degree $2^{m} \geq 4$ and how we compute their associated characters. We use the factorization $\chi_{N}=\prod_{q \mid f} \chi_{q}$ corresponding to the decomposition $f_{N}=\prod_{q \mid f} q^{\nu_{q}(f)}$

1.1. Cyclic quartic fields. We notice that a quartic character of conductor a $q$-power has conductor 16 or an odd prime $q \equiv 1(\bmod 4)$. Conversely, whenever $q \equiv 1(\bmod 4)$ is prime, we let $\chi_{q}^{(2)}$ denote the quartic character of conductor $q$ defined by means of

$$
\chi_{q}^{(2)}\left(a_{q}\right)=i=\exp (2 \pi i / 4) \quad \text { where } a_{q}=\min \left\{a \geq 1 ;\left(\frac{a}{q}\right)=-1\right\}
$$

(where $\left(\frac{n}{q}\right)$ denotes Legendre's symbol). Hence, $\chi_{q}^{(2)}(-1)=(-1)^{(q-1) / 4}$. To compute the values of $\chi_{q}^{(2)}$ we determine $a_{q}$ and $b_{q}=a_{q}^{(q-1) / 4}$. Then, for each $n$ coprime with $q$, we have

$$
\chi_{q}^{(2)}(n)=i^{k} \quad \text { where } k=\min \left\{x \geq 0 ; n^{(q-1) / 4} \equiv b_{q}^{x} \quad(\bmod q)\right\} .
$$

Note that $\chi_{q}^{(2)}$ and its conjugate $\overline{\chi_{q}^{(2)}}$ are the only quartic characters of conductor $q$. We also let $\chi_{16}^{+}$denote the even quartic character of conductor 16 defined by the following table:

\begin{tabular}{|l|llllllll|}
\hline$n$ & 1 & 3 & 5 & 7 & 9 & 11 & 13 & 15 \\
\hline$\chi_{16}^{+}$ & 1 & $-i$ & $i$ & -1 & -1 & $i$ & $-i$ & 1 \\
\hline
\end{tabular}

Set $\chi_{16}^{-}(n)=\left(\frac{-4}{n}\right) \chi_{16}^{+}(n)$. We notice that $\chi_{16}^{ \pm}$and their conjugates $\overline{\chi_{16}^{ \pm}}$are the only primitive quartic characters of 2 -power conductors. Since $\overline{\chi_{q}^{(2)}}(n)=\left(\frac{n}{q}\right) \chi_{q}^{(2)}(n)$, we easily get:

Proposition 3. Let $N$ be a cyclic quartic field. Then $f_{N}=2^{k} f_{1} f_{2}$ with $k \in$ $\{0,2,3,4\}$, whith $f=f_{1} f_{2}$ odd and square-free, and $f_{2}>1$ such that $q \mid f_{2}$ implies $q \equiv 1(\bmod 4)$. Conversely, for such an $f_{N}$ we have a bijective correspondence 
between the cyclic quartic fields $N$ of conductor $f_{N}$ and the following quartic characters of conductor $f_{N}$ :

\begin{tabular}{|c|l|l|l|l|l|}
\hline$k$ & $\chi_{N}(n)$ & $f_{N^{+}}$ & $\chi_{N}(-1)$ & $g_{N}$ & $d_{2}$ \\
\hline 0 & $\left(\frac{n}{f_{1} d_{2}}\right) \prod_{q \mid f_{2}} \chi_{q}^{(2)}(n)$ & $f_{2}$ & $(-1)^{\frac{f_{1}-1}{2}+\frac{f_{2}-1}{4}}$ & $2^{r} 4^{s-1}$ & $1 \leq d_{2}<\sqrt{f_{2}}$ \\
\hline 2 & $\left(\frac{-4}{n}\right)\left(\frac{n}{f_{1} d_{2}}\right) \prod_{q \mid f_{2}} \chi_{q}^{(2)}(n)$ & $f_{2}$ & $-(-1)^{\frac{f_{1}-1}{2}+\frac{f_{2}-1}{4}}$ & $2^{r+1} 4^{s-1}$ & $1 \leq d_{2}<\sqrt{f_{2}}$ \\
\hline 3 & $\left(\frac{ \pm 8}{n}\right)\left(\frac{n}{f_{1} d_{2}}\right) \prod_{q \mid f_{2}} \chi_{q}^{(2)}(n)$ & $f_{2}$ & $\pm(-1)^{\frac{f_{1}-1}{2}+\frac{f_{2}-1}{4}}$ & $2^{r+1} 4^{s-1}$ & $1 \leq d_{2}<\sqrt{f_{2}}$ \\
\hline 4 & $\begin{array}{c}\left(\frac{ \pm 4}{n}\right)\left(\frac{n}{f_{1} d_{2}}\right) \chi_{16}^{+}(n) \\
\end{array}$ & $8 f_{2}$ & $\pm(-1)^{\frac{f_{1}-1}{2}}+\frac{f_{2}-1}{4}$ & $2^{r} 4^{s}$ & $1 \leq d_{2} \leq f_{2}$ \\
\hline
\end{tabular}

Here, $r$ and $s$ denote the number of prime divisors of $f_{1}$ and $f_{2}$, respectively, $d_{2}$ denotes a divisor of $f_{2}$ and when $d$ is positive and odd, we let $\left(\frac{n}{d}\right)$ denote the Jacobi's symbol.

1.2. Cyclic fields of 2-power degrees. Now, let us describe the primitive characters of any given 2-power order $2^{m} \geq 4$. Whenever $q$ is an odd prime we set

$$
j_{q}=\max \left\{j \geq 1 ; 2^{j} \mid q-1\right\}, m_{q}=\min \left(j_{q}, m\right) \text { and } a_{q}=\min \left\{a \geq 1 ;\left(\frac{a}{q}\right)=-1\right\}
$$

and let $\chi_{q}^{(m)}$ be the character of conductor $q$ and order $2^{m_{q}}$ well defined by $\chi_{q}^{(m)}\left(a_{q}\right)$ $=\exp \left(2 \pi i / 2^{m_{q}}\right)$. Hence,

$$
\chi_{q}^{(m)}(-1)= \begin{cases}1 & \text { if } j_{q}>m, \\ -1 & \text { if } j_{q} \leq m .\end{cases}
$$

We note that any character of conductor $q$ and order dividing $2^{m}$ has order dividing $2^{m_{q}}$ and is a power of $\chi_{q}^{(m)}$. To compute the values of $\chi_{q}^{(m)}$ we determine $a_{q}$ and $b_{q}=a_{q}^{(q-1) / 2^{m_{q}}}$. Then, for each $n$ coprime with $q$, we have

$$
\chi_{q}^{(m)}(n)=\exp \left(2 k \pi i / 2^{m_{q}}\right) \quad \text { where } k=\min \left\{x \geq 0 ; n^{(q-1) / 2^{m_{q}}} \equiv b_{q}^{x} \quad(\bmod q)\right\} .
$$

Finally, for $k \geq 3$ we let $\chi_{2}^{(k)}$ be the even primitive character of conductor $2^{k}$ and order $2^{k-2}$ well defined by means of $\chi_{2}^{(k)}(-1)=1$ and $\chi_{2}^{(k)}(5)=\exp \left(2 \pi i / 2^{k-2}\right)$. Any primitive character $\chi$ of conductor $2^{k}$ has order $2^{k-2}$ and there exists $n_{0}$ odd such that $\chi(n)=\left(\frac{ \pm 4}{n}\right)\left(\chi_{2}^{(k)}(n)\right)^{n_{0}}$.

Proposition 4. Let $N$ be a cyclic field of degree $2^{m} \geq 4$. Then $f_{N}=2^{k} f$ with $k \neq 1,0 \leq k \leq m+2$ and with $f$ odd and square-free and such that if $k<m+2$, then at least one of the prime divisors of $f$ satisfies $q \equiv 1\left(\bmod 2^{m}\right)$. Conversely, for such an $f_{N}$ we have a bijective correspondence between the cyclic fields $N$ of degree $2^{m}$ and conductor $f_{N}$ and the following characters of order $2^{m}$ and conductor $f_{N}$ :

\begin{tabular}{|l|l|l|l|}
\hline Case & $k$ & $\chi_{N}(n)$ & $g_{N}$ \\
\hline 1 & $k=0$ & $\prod_{q \mid f}\left(\chi_{q}^{(m)}\right)^{n_{q}}$ & $2^{-m} \prod_{q \mid f} e_{q}$ \\
\hline 2 & $k=2$ & $\left(\frac{-4}{n}\right) \prod_{q \mid f}\left(\chi_{q}^{(m)}\right)^{n_{q}}$ & $2^{1-m} \prod_{q \mid f} e_{q}$ \\
\hline 3 & $3 \leq k<m+2$ & $\left(\frac{ \pm 4}{n}\right)\left(\chi_{2}^{(k)}(n)\right)^{n_{0}} \prod_{q \mid f}\left(\chi_{q}^{(m)}\right)^{n_{q}}$ & $2^{k-2-m} \prod_{q \mid f} e_{q}$ \\
\hline 4 & $k=m+2$ & $\left(\frac{ \pm 4}{n}\right) \chi_{2}^{(k)}(n) \prod_{q \mid f}\left(\chi_{q}^{(m)}\right)^{n_{q}}$ & $\prod_{q \mid f} e_{q}$ \\
\hline
\end{tabular}

Here, we have $1 \leq n_{0} \leq 2^{k-2}, n_{0}$ odd, $1 \leq n_{q} \leq 2^{m_{q}}-1$ and $e_{q}=2^{m_{q}} / \operatorname{gcd}\left(2^{m_{q}}, n_{q}\right)$ is the order of the component $\left(\chi_{q}^{(m)}\right)^{n_{q}}$ of $\chi_{N}$. Hence, in cases 1, 2 and 3, at least 
one of the $e_{q}$ 's is equal to $2^{m}$, and we assume that $n_{q}=1$ for the greatest odd prime $q$ such that $e_{q}=2^{m}$. Note that (2) enables us to compute $\chi_{N}(-1)$.

\section{LOWER BOUNDS ON RELATIVE CLASS NUMBERS OF NONQUADRATIC IMAGINARY CYCLIC QUARTIC FIELDS}

We give reasonable upper bounds on the degrees and conductors of the nonquadratic imaginary cyclic fields of 2-power degrees whose class numbers are equal to their genus numbers.

Theorem 5 (See [Lou3]). Let $N$ be an imaginary cyclic field of degree $2 n=2^{m} \geq$ 4 and conductor $f$. Choose $\epsilon_{f}=1-\left(2 \pi n e^{1 / n} / \sqrt{f}\right)$ or $\epsilon_{f}=\frac{2}{5} \exp (-2 \pi n / \sqrt{f})$. We have

$$
h_{N}^{-} \geq \frac{2 \epsilon_{f}}{e(2 n-1)}\left(\frac{f}{\pi^{2}(\log f+0.05)^{2}}\right)^{n / 2} .
$$

Corollary 6. Let $N$ be an imaginary cyclic field of degree $2 n=2^{m} \geq 4$ and conductor $f$. Assume $h_{N}=g_{N}$. Then $2 n=4$ implies $f \leq 210000,2 n=8$ implies $f \leq 14000,2 n \geq 16$ implies $f \leq 3800$ and $2 n \geq 32$ implies $f \leq 1800$.

Proof. Let $t$ be the number of prime divisors of $f$. Set $f_{t}=\prod_{i=1}^{t} p_{i}$ where $p_{1}=3$, $p_{2}=4$ and $\left(p_{i}\right)_{i \geq 3}$ is the increasing sequence of all the odd primes greater than or equal to 5. If $h_{N}=g_{N}$, then $h_{N}^{-}=2^{t-1}$ (Lemma 2) and $f \geq f_{t}$. First, assume $N$ is quartic. Using (3) with the choice $\epsilon_{f}=1-\left(2 \pi n e^{1 / n} / \sqrt{f}\right)=1-4 \pi \sqrt{e / f}$, we get

$$
2^{t-1} \geq \epsilon_{f_{t}} \frac{2 f_{t}}{3 e \pi^{2}\left(\log f_{t}+0.05\right)^{2}}
$$

which implies $t \leq 6, h_{N}^{-} \leq 2^{5}$ and using (3) once again yields

$$
2^{5} \geq \epsilon_{f} \frac{2 f}{3 e \pi^{2}(\log f+0.05)^{2}}
$$

hence implies $f \leq 210000$. In the same way, if $N$ is octic, then we get $t \leq 5$, $h_{N}^{-} \leq 16$ and $f \leq 14000$. Now, if $2 n=2^{m}=[N: Q] \geq 16$, then we choose $\epsilon_{f}=\frac{2}{5} \exp (-2 \pi n / \sqrt{f})$. We get $t \leq 4, h_{N}^{-} \leq 8$ and the desired results.

\section{Conclusion and tables}

First, we use Propositions 3 and 4 to find all the non-quadratic imaginary cyclic fields of 2-power degrees with conductors less than or equal to the bounds given in Corollary 6. Second, we use (1) to compute the relative class numbers of all these fields and we disregard those which do not satisfy the necessary condition for $h_{N}=g_{N}$ given in Lemma 2. This provides us with a short list of 28 nonquadratic imaginary cyclic number fields of 2-power degrees containing all the nonquadratic imaginary cyclic number fields of 2-power degrees with class numbers equal to their genus class numbers. Third, we use Propositions 3 and 4 to compute the genus class numbers $g_{N}$ of these 28 fields $N$. To prove Theorem 1 , it finally remains to compute the class numbers $h_{N^{+}}$of the maximal real subfields $N^{+}$of the 28 fields $N$ of this list and to check whether $h_{N}=g_{N}$ holds, which holds for the 28 occurrences of this short list. The following three tables sum up the results of our numerical computations (we let $C_{2^{k}}$ denote a cyclic group of order $2^{k}$ and $\mathcal{H}_{N}$ denote the ideal class group of $N)$ : 
TABLE 1 . The quartic case

\begin{tabular}{|l|l|l|l|l|l|l|l|l|}
\hline Index & $f_{N}$ & $f_{N^{+}}$ & $g_{N}$ & $h_{N}^{-}$ & $h_{N^{+}}$ & $h_{N}$ & $N$ & $\mathcal{H}_{N}$ \\
\hline 1 & 5 & 5 & 1 & 1 & 1 & 1 & $Q(\sqrt{-(5+2 \sqrt{5})})$ & $\{1\}$ \\
2 & 13 & 13 & 1 & 1 & 1 & 1 & $Q(\sqrt{-(13+2 \sqrt{13})})$ & $\{1\}$ \\
3 & 16 & 8 & 1 & 1 & 1 & 1 & $Q(\sqrt{-(2+\sqrt{2})})$ & $\{1\}$ \\
4 & 29 & 29 & 1 & 1 & 1 & 1 & $Q(\sqrt{-(29+2 \sqrt{29})})$ & $\{1\}$ \\
5 & 37 & 37 & 1 & 1 & 1 & 1 & $Q(\sqrt{-(37+6 \sqrt{37})})$ & $\{1\}$ \\
6 & 40 & 5 & 2 & 2 & 1 & 2 & $Q(\sqrt{-(5+\sqrt{5})})$ & $C_{2}$ \\
7 & 48 & 8 & 2 & 2 & 1 & 2 & $Q(\sqrt{-3(2+\sqrt{2})})$ & $C_{2}$ \\
8 & 53 & 53 & 1 & 1 & 1 & 1 & $Q(\sqrt{-(53+2 \sqrt{53})})$ & $\{1\}$ \\
9 & 60 & 5 & 4 & 4 & 1 & 4 & $Q(\sqrt{-3(5+2 \sqrt{5})})$ & $C_{2} \times C_{2}$ \\
10 & 61 & 61 & 1 & 1 & 1 & 1 & $Q(\sqrt{-(61+6 \sqrt{61})})$ & $\{1\}$ \\
11 & 65 & 5 & 2 & 2 & 1 & 2 & $Q(\sqrt{-13(5+2 \sqrt{5})})$ & $C_{2}$ \\
12 & 65 & 13 & 2 & 2 & 1 & 2 & $Q(\sqrt{-5(13+2 \sqrt{13})})$ & $C_{2}$ \\
13 & 80 & 8 & 2 & 2 & 1 & 2 & $Q(\sqrt{-5(2+\sqrt{2})})$ & $C_{2}$ \\
14 & 80 & 40 & 4 & 2 & 2 & 4 & $Q(\sqrt{-(10+3 \sqrt{10})})$ & $C_{4}$ \\
15 & 85 & 5 & 2 & 2 & 1 & 2 & $Q(\sqrt{-17(5+2 \sqrt{5})})$ & $C_{2}$ \\
16 & 85 & 85 & 4 & 2 & 2 & 4 & $Q(\sqrt{-(85+6 \sqrt{85})})$ & $C_{4}$ \\
17 & 104 & 13 & 2 & 2 & 1 & 2 & $Q(\sqrt{-(13+3 \sqrt{13})})$ & $C_{2}$ \\
18 & 105 & 5 & 4 & 4 & 1 & 4 & $Q(\sqrt{-21(5+2 \sqrt{5})})$ & $C_{2} \times C_{2}$ \\
19 & 119 & 17 & 2 & 2 & 1 & 2 & $Q(\sqrt{-7(17+4 \sqrt{17})})$ & $C_{2}$ \\
20 & 120 & 5 & 4 & 4 & 1 & 4 & $Q(\sqrt{-3(5+\sqrt{5})})$ & $C_{2} \times C_{2}$ \\
21 & 140 & 5 & 4 & 4 & 1 & 4 & $Q(\sqrt{-(7(5+2 \sqrt{5})})$ & $C_{2} \times C_{2}$ \\
22 & 195 & 65 & 8 & 4 & 2 & 8 & $Q(\sqrt{-3(65+8 \sqrt{65})})$ & $C_{2} \times C_{4}$ \\
23 & 255 & 17 & 4 & 4 & 1 & 4 & $Q(\sqrt{-15(17+4 \sqrt{17})})$ & $C_{2} \times C_{2}$ \\
\hline
\end{tabular}

TABle 2. The octic case

\begin{tabular}{|l|l|l|l|l|l|l|l|l|l|}
\hline Index & $f_{N}$ & $f_{N^{+}}$ & $g_{N}$ & $h_{N}^{-}$ & $N$ & $N^{+}$ & $h_{N^{+}}$ & $h_{N}$ & $\mathcal{H}_{N}$ \\
\hline 24 & 32 & 16 & 1 & 1 & $Q(\sqrt{-(2+\sqrt{2+\sqrt{2}})})$ & $Q(\sqrt{2+\sqrt{2}})$ & 1 & 1 & $\{1\}$ \\
25 & 41 & 41 & 1 & 1 & $N \leftrightarrow \chi_{41}^{(3)}$ & $Q(\sqrt{41+4 \sqrt{41}})$ & 1 & 1 & $\{1\}$ \\
26 & 51 & 17 & 2 & 2 & $N \leftrightarrow \chi_{3}^{(3)} \chi_{17}^{(3)}$ & $Q(\sqrt{17+4 \sqrt{17}})$ & 1 & 2 & $C_{2}$ \\
27 & 85 & 85 & 4 & 2 & $N \leftrightarrow\left(\chi_{5}^{(3)}\right)^{3} \chi_{17}^{(3)}$ & $Q(\sqrt{5(17+4 \sqrt{17})})$ & 2 & 4 & $C_{4}$ \\
\hline
\end{tabular}


TABLE 3 . The degree $\geq 16$ case

\begin{tabular}{|l|l|l|l|l|l|l|}
\hline Index: 28 & $f_{N}=f_{N^{+}}=17$ & $h_{N}^{-}=1$ & $N=Q\left(\zeta_{17}\right)$ & $N^{+}=Q\left(\cos \left(\frac{2 \pi}{17}\right)\right.$ & $h_{N}=1$ & $\mathcal{H}_{N}=\{1\}$ \\
\hline
\end{tabular}

Our computations agree with those done in [PK], [Yam], [Lou1] and [Lou3]. In particular, according [Lou3, Proposition 5] the 22 nonquadratic imaginary cyclic number fields of 2-power degrees with cyclic ideal class groups of 2-power orders found in [Lou3] also appear in our present determination. Note that our present paper, [Lou1] and [Lou3] provide us with the solutions of the following three class groups problems for nonquadratic imaginary cyclic number fields of 2-power degrees: 1) the exponent 2 class group problem, 2) the cyclic class group of 2-power order problem, and 3) the one class in each genus problem.

\section{REFERENCES}

[Ham] M. Hamamura. On the absolute class fields of certain algebraic number fields. Nat. Sci. Rep. Ochanomizu Univ., 32 (1981), 23-34. MR 83a:12014

[Lou1] S. Louboutin. Determination of all nonquadratic imaginary cyclic number fields of 2-power degrees with ideal class groups of exponents $\leq 2$. Math. Comp., 64 (1995), 323-340. MR 95c: 11124

[Lou2] S. Louboutin. A finiteness theorem for imaginary abelian number fields. Manuscripta math., 91 (1996), 343-352. MR 97f:11089

[Lou3] S. Louboutin. CM-fields with cyclic ideal class groups of 2-power orders. J. of Nb. Th., 67 (1997), 1-10. MR 98h:11139

[Miy] I. Miyada. On imaginary abelian number fields of type $(2,2, \cdots, 2)$ with one class in each genus. Manuscripta math., 88 (1995), 535-540. MR 96j:11146

[PK] Y.-H. Park and S.-H. Kwon. Determination of all non-quadratic imaginary cyclic number fields of 2-power degree with class number $\leq 20$. Acta Arith., 83 (1998), 211-223. CMP 98:09

[Wa] L.C. Washington. Introduction to Cyclotomic Fields. Springer-Verlag, Grad. Texts Math. 83 (1997). MR 85g:11001

[Yam] K. Yamamura. The determination of the imaginary abelian number fields with classnumber one. Math. Comp., 62 (1994), 899-921. MR 94g:11096

Université de Caen, UfR Sciences, Département de Mathématiques, 14032 Caen cedex, FRANCE

E-mail address: loubouti@math.unicaen.fr 